\title{
Frustration in a Mixed-Spin Ising Model With Multi-Spin Interactions
}

\author{
M. JAŠČUR ${ }^{a, *}$, V. ŠTUBŇA ${ }^{a}$, K. SZALOWSKI ${ }^{b}$, T. BALCERZAK ${ }^{b}$ \\ ${ }^{a}$ Institute of Physics, Faculty of Sciences, P.J. Šafárik University in Košice \\ Park Angelinum 9, 04154 Košice, Slovakia \\ ${ }^{b}$ Department of Solid State Physics, University of Łódź, ul. Pomorska 149/153, 90-236 Łódź, Poland
}

\begin{abstract}
In this work we investigate a mixed spin-1/2 and spin-1 Ising model on a decorated square lattice. In addition to standard pair interactions between nearest neighbors, we also consider in our calculations three-site four-spin interactions and the effect of single ion anisotropy. Applying the well-known decoration-iteration transformation, we derive a simple relation between the partition function of the model under investigation and the Onsager's partition function for the simple square lattice. With the help of this mapping relation we are able to calculate exact expressions for all relevant physical quantities of the system (for example, the magnetization, internal energy, Gibbs free energy, entropy and specific heat). The most interesting results have been found in the model without pair interactions. In this case, besides the standard ordered and disordered phases, there also appears an interesting phase which is ordered only partially. Analyzing relevant physical quantities of the system, we have found that the partial ordering is closely related to very strong frustrations that lead to non-zero entropy at $T=0$.
\end{abstract}

DOI: 10.12693/APhysPolA.126.48

PACS: $5.50 .+\mathrm{q}, 75.10 \mathrm{Hk}$

\section{Introduction}

Theoretical investigation of many-body interactions represents very complex mathematical problem. For that reason, the interaction terms in many-body systems are usually limited to pair-interactions only. On the other hand, the magnetic systems described by Ising or Heisenberg models represent rare examples of physical systems, where many-body interactions are mathematically tractable. Understanding of higher-order interactions is important for the theory of magnetism, as well as for the fundamental understanding of many-body interactions. This study deals with the Ising model including the special, so-called, three-site four-spin interactions among atoms [1].

\section{Formulation}

We will investigate a mixed-spin Ising model on a decorated square lattice. The Hamiltonian of the system is given by

$$
H=\sum_{k=1}^{2 N} H_{k},
$$

where $N$ denotes the number of spins on undecorated lattice and the summation runs over all $2 N$ bonds (or $2 N$ decorating spins). The Hamiltonian $H_{k}$ includes all interactions terms related to the $k$-th decorating atom and can be expressed in the form

$$
H_{k}=-J S_{k}\left(\mu_{k 1}+\mu_{k 2}\right)-J_{4} S_{k}^{2} \mu_{k 1} \mu_{k 2}-D S_{k}^{2} .
$$

Here $\mu_{k i}= \pm 1 / 2, S_{k}=0, \pm 1, J$ and $J_{4}$ denote the

\footnotetext{
* corresponding author; e-mail: Michal.Jascur@upjs.sk
}

pair and three-site four-spin exchange interactions, respectively and $D$ represents a single-ion anisotropy parameter [1]. The partition function of the decorated system is given by

$$
Z=\sum_{\left\{\mu_{k i}= \pm \frac{1}{2}\right\}} \sum_{\left\{S_{k=0, \pm 1}\right\}} \exp (-\beta H) .
$$

Substituting (2) into (3) one obtains

$$
Z=\sum_{\left\{\mu_{k i}= \pm \frac{1}{2}\right\}} \prod_{k=1}^{2 N} \sum_{S_{k}=0, \pm 1} \exp \left(-\beta H_{k}\right) .
$$

After introducing the decoration-iteration transformation [2]

$$
\sum_{S_{k}=0, \pm 1} \exp \left(-\beta H_{k}\right)=A \exp \left(\beta R \mu_{k 1} \mu_{k 2}\right)
$$

we obtain the following simple relation between partition functions of decorated and undecorated system

$$
Z=A^{2 N} Z_{0}(\beta R)
$$

One should emphasize here that relation (6) is exact, because the partition function $Z_{0}$ of the simple (i.e. undecorated) square lattice is well-known (see $[2,3]$ and references therein). The parameters $A$ and $R$ can be very simply calculated from the Eq. (5). Indeed, performing summation in Eq. 5 and setting all possible values of $\mu_{k 1}$ and $\mu_{k 2}$ we obtain expressions:

$$
\begin{aligned}
& A=\sqrt{V_{1} V_{2}}, \quad \beta R=2\left(\ln V_{1}-\ln V_{2}\right), \\
& V_{1}=1+2 \exp \left(\beta D+\beta J_{4} / 4\right) \cosh (\beta J), \\
& V_{2}=1+2 \exp \left(\beta D-\beta J_{4} / 4\right) .
\end{aligned}
$$

Eqs. (6)-(9) enable exact calculation of phase diagrams and all relevant thermodynamic quantities. 


\section{Numerical results}

In this part we will show some interesting results for the system under investigation. In order to simplify notations, we have introduced the reduced dimensionless quantities, namely $\alpha=J / J_{4}$ and $d=D / J_{4}$. As usually, we have at first established the ground-state phase diagram of our system in $\alpha-d$ space. The numerical results are depicted in Fig. 1.

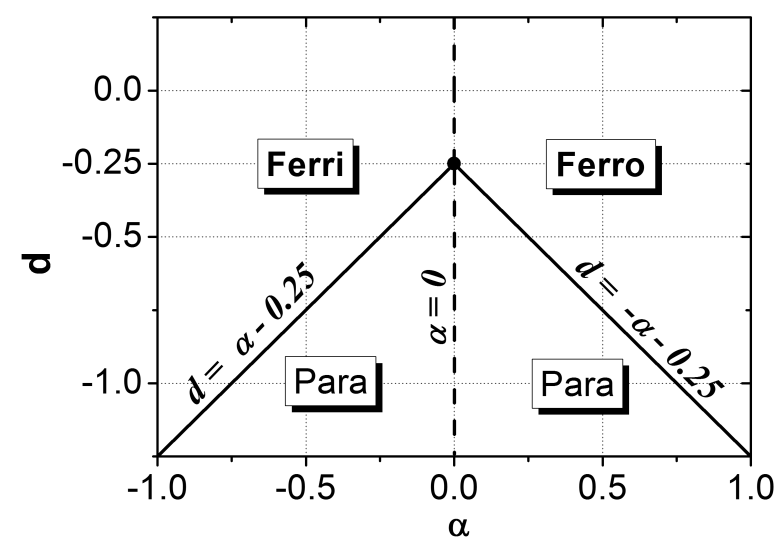

Fig. 1. The ground-state phase diagram in $\alpha-d$ space.

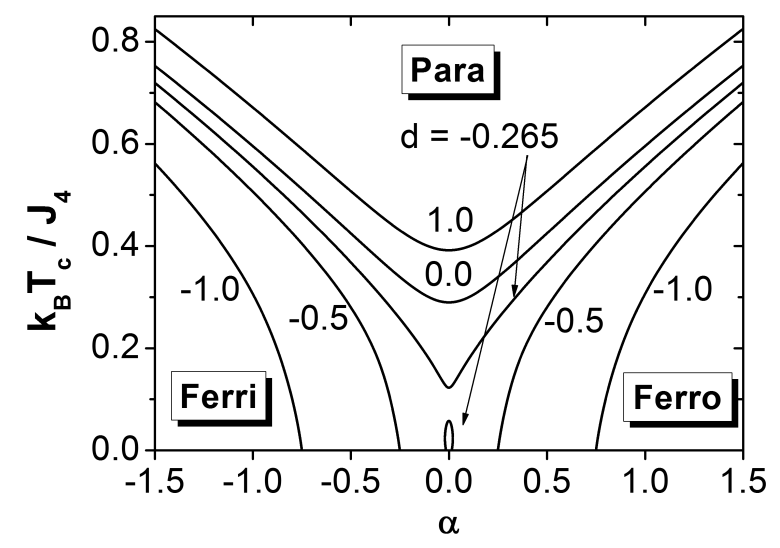

Fig. 2. Phase diagram in $\alpha-T_{c}$ space.

The full line $d=-|\alpha|-0.25$ separates the disordered paramagnetic phase from the ordered ferro- or ferrimagnetic phases. The dashed line $\alpha=0$ represents a special line in phase space along which one finds three different frustrated phases with nonzero entropy at $T=0$. Our numerical analysis reveals that $S / N=k_{B} \ln 4$ for $d>-0.25, S / N=k_{B} \ln 2$ for $d<-0.25$ and finally, $S / N=2.21 k_{B}$ for $d=-0.25$. We have also investigated the sublattice magnetizations of the system and several correlation functions at $T=0$ and we have found that in the case of $d>-0.25$ all decorating atoms occupy the spin states \pm 1 with equal probability, while all other atoms prefer the spin $1 / 2$ state. On the other hand, for $d<-0.25$ all decorating atoms prefer the spin 0 state, while all other atoms occupy equally the spin states $\pm 1 / 2$. Finally, one should notice that the strongest frustrations appear for the system with $d=-0.25$, however, the spin configurations cannot be described so simply as in previous cases.

In order to understand magnetic properties of the system, we have also calculated finite-temperature phase diagrams. Our results are shown in Fig. 2, where we have plotted the critical temperature as a function of the parameter $\alpha$ for several fixed values of $d$. As one can see from the figure, the critical temperature of the system is independent of the sign of pair exchange interaction and the system is ordered below each curve and disordered when the temperature becomes high enough. Moreover, it is also evident that low-temperature behaviour of the critical temperatures is fully in agreement with the ground-state phase diagram.

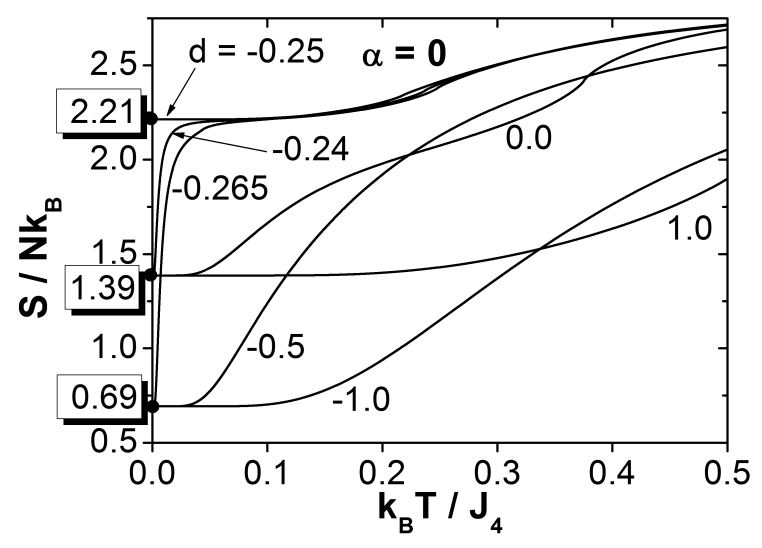

Fig. 3. Thermal variations of the reduced entropy.

Finally, in order to study some properties of frustrated phases of the system, we have calculated temperature variations of the reduced entropy of the system. The results depicted in Fig. 3 agree with our previous analysis of possible spin configurations at $T=0$.

\section{Conclusions}

In this work we have studied some interesting properties of the exactly solvable mixed spin-1/2 and spin-1 Ising model with pair and three-site four-spin interactions. We have mainly discussed the ground-state and finite-temperature phase diagrams on decorated square lattice. The presented results clearly indicate that in the system with zero pair exchange interaction there exist three different frustrated phases with finite values of the entropy at $T=0$.

\section{Acknowledgments}

This work was supported by the project VEGA No. $1 / 0234 / 12$.

\section{References}

[1] T. Iwashita, N. Uryu, J. Phys. C 17, 855 (1984).

[2] J. Strečka, Phys. Lett. A 374, 3718 (2010).

[3] S. Lacková, M. Jaščur, Phys. Rev. E 64, 036126 (2001). 